\title{
Minimal Criteria for Lung Ultrasonography in Internal Medicine
}

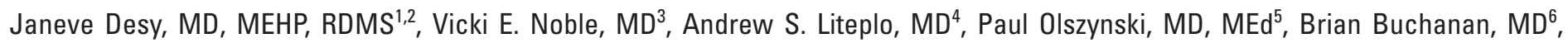
Renee K. Dversdal, $\mathrm{MD}^{7}$, Shane Arishenkoff, MD ${ }^{8}$, Gigi Liu, MD, MSc ${ }^{9}$, Elaine Dumoulin, MD ${ }^{10}$, Irene W. Y. Ma, MD, PhD, RDMS, RDCS ${ }^{1,2,4}$

\begin{abstract}
${ }^{1}$ Division of General Internal Medicine, Department of Medicine, University of Calgary, Calgary, AB, Canada; ${ }^{2}$ W21C, University of Calgary, Calgary, AB, Canada; ${ }^{3}$ Department of Emergency Medicine, University Hospitals, Cleveland Medical Center, Case Western Reserve School of Medicine, Cleveland, OH, USA; ${ }^{4}$ Division of Emergency Ultrasound, Department of Emergency Medicine, Massachusetts General Hospital, Boston, Harvard Medical School, Boston, MA, USA; ${ }^{5}$ Department of Emergency Medicine, University of Saskatchewan, Saskatoon, SK, Canada; ${ }^{6}$ Department of Critical Care, University of Alberta, Edmonton, AB, Canada; ${ }^{7}$ Department of Medicine, Oregon Health \& Science University, Portland, OR, USA; ${ }^{8}$ Division of General Internal Medicine, Department of Medicine, University of British Columbia, Vancouver, BC, Canada; ${ }^{9}$ Department of Medicine, Johns Hopkins University School of Medicine, Baltimore, MD, USA;

${ }^{10}$ Division of Respiratory Medicine, Department of Medicine, University of Calgary, Calgary, AB, Canada
\end{abstract}

Author for correspondence: Janeve Desy: janeve.desy@ucalgary.ca

Received: 11 December 2020; Accepted after revision: 5 February 2021; Published: 21 June 2021

DOI https://doi.org/10.22374/cjgim.v16i2.507

\begin{abstract}
Background

Point-of-care lung ultrasound (LUS) examination is increasingly utilized in Internal Medicine. To improve the standardization of LUS education and clinical use, explicit minimal criteria for defining what is an acceptable and clinically useful image are needed.
\end{abstract}

\section{Methods}

A 97-item online survey of potential minimal criteria for common uses of LUS in Internal Medicine was developed and sent to 10 international point-of-care ultrasound experts. Their opinion on the inclusion of each item was sought and items not achieving consensus (defined as agreement by at least $70 \%$ of the experts) were reassessed in subsequent rounds. A total of three rounds were conducted.

\section{Results}

Seventy-four minimal criteria were agreed upon for inclusion, 24 were agreed upon for exclusion, and two did not reach consensus.

\section{Conclusions}

Experts agreed on 74 minimal criteria for Internal Medicine LUS. The use of these minimal criteria during teaching and clinical use is strongly recommended.

\section{Résumé \\ Contexte}

Léchographie pulmonaire au point d'intervention est de plus en plus utilisée en médecine interne. Pour améliorer l'uniformisation de la formation sur léchographie pulmonaire et de 
son utilisation clinique, il faut des critères minimaux explicites pour définir ce qu'est une image acceptable et utile sur le plan clinique.

\section{Méthodologie}

Un sondage en ligne de 97 éléments portant sur des critères minimaux possibles dans l'utilisation courante de l'échographie pulmonaire en médecine interne a été élaboré et soumis à 10 experts internationaux en échographie au point d'intervention. Leur avis sur l'inclusion de chaque élément a été sondé, et les éléments pour lesquels il n’y avait pas de consensus (défini par l’accord d’au moins $70 \%$ des experts) ont été réévalués lors de tours suivants. Au total, trois tours ont été effectués.

\section{Résultats}

Soixante-quatorze critères minimaux ont été acceptés, 24 ont été exclus et deux nont pas fait consensus.

\section{Conclusions}

Les experts se sont entendus sur 74 critères minimaux relatifs à l'échographie pulmonaire en médecine interne. L'utilisation de ces critères minimaux au cours de l'enseignement et de l'utilisation clinique est fortement recommandée.

\section{Background}

The role of point-of-care ultrasound (POCUS) in the care of medical patients is being increasingly recognized. ${ }^{1-3}$ Of the recommended Canadian Internal Medicine POCUS applications, ${ }^{3}$ lung ultrasound (LUS) is one of the easiest to learn and has high clinical utility. ${ }^{4}$ Its use in medical patients with dyspnea, for example, is associated with increased diagnostic accuracy at the bedside. ${ }^{5-7}$ Its use may also be gaining importance in the assessment of patients with coronavirus-19. ${ }^{8}$ Despite it being relatively easy to learn, to support the use of LUS clinically, appropriate training in image acquisition and interpretation is required. It is generally accepted by POCUS experts that images not meeting minimal criteria, such as those that have insufficient depth or inadequate optimization of the pleural line, should not be used to support clinical decision-making, ${ }^{9}$ as they may introduce serious diagnostic errors. Both achieving minimal criteria during image acquisition and the recognition of when these criteria have not been met are fundamental to POCUS competency. ${ }^{9}$ In addition, the use of minimal criteria in POCUS training programs is considered a key indicator of high-quality education. ${ }^{10}$

Despite the critical importance of minimal criteria, existing resources on learning LUS, while useful, focus primarily on image acquisition and interpretation, ${ }^{4,11,12}$ without an explicit list of minimal criteria that an image must meet. As POCUS education expands across the country, articulation of such criteria becomes increasingly important if high-quality education is to be standardized. Given that trained POCUS experts agree on the importance of minimal criteria, ${ }^{9}$ through consensus methods, this study seeks to encapsulate this codex of expert knowledge into an explicit list of minimal criteria for LUS. These minimal criteria can then be used to guide clinical use as well as LUS education for residency training programs.

\section{Methods \\ Survey Development}

This study received approval from the Conjoint Health Research Ethics Board at the University of Calgary (\#REB16-2452). Using existing key reference resources for LUS, ${ }^{11,13-22}$ two independent investigators (J.D. and I.M.) drafted a list of minimal criteria for all intended applications of LUS, to be as inclusive as possible. Disagreements were resolved by discussion and if necessary consensus from additional investigators. This 78-item list was formatted into an online survey (www.surveymonkey.com, SurveyMonkey Inc., San Mateo, CA, USA).

This draft online survey was piloted in May 2019 on 14 Internal Medicine and Family Medicine physicians with at least 1 month of formal ultrasound training for feedback on survey length, clarity, response options, missing or redundant items, and flow. Feedback was reviewed by both J.D. and I.M. and incorporated into the final 97-item survey. 
Table 1. Baseline Characteristics of the 10 Participants on the Expert Panel

\begin{tabular}{|c|c|}
\hline Characteristic & $\mathbf{N}(\%)$ \\
\hline \multicolumn{2}{|l|}{ Country of Practice } \\
\hline Canada & $5(50)$ \\
\hline USA & $5(50)$ \\
\hline \multicolumn{2}{|l|}{ Specialty } \\
\hline Emergency medicine & $3(30)$ \\
\hline Critical care medicine & $1(10)$ \\
\hline General internal medicine & $6(60)$ \\
\hline \multicolumn{2}{|l|}{ Gender } \\
\hline Male & $4(40)$ \\
\hline Female & $6(60)$ \\
\hline \multicolumn{2}{|l|}{ Years of experience using POCUS* } \\
\hline $3-4$ & $1(10)$ \\
\hline $5-6$ & $3(30)$ \\
\hline $7-8$ & $1(10)$ \\
\hline $9-10$ & $1(10)$ \\
\hline$>10$ & $4(40)$ \\
\hline \multicolumn{2}{|l|}{ Years of experience teaching POCUS* } \\
\hline $1-2$ & $1(10)$ \\
\hline $3-4$ & $2(20)$ \\
\hline $5-6$ & $1(10)$ \\
\hline $7-8$ & $2(20)$ \\
\hline $9-10$ & $1(10$ \\
\hline$>10$ & $3(30)$ \\
\hline \multicolumn{2}{|l|}{ Years of experience assessing POCUS* } \\
\hline $1-2$ & $2(20)$ \\
\hline $3-4$ & $1(10)$ \\
\hline $5-6$ & $2(20)$ \\
\hline $7-8$ & $2(20)$ \\
\hline $9-10$ & $1(10)$ \\
\hline$>10$ & $2(20)$ \\
\hline \multicolumn{2}{|l|}{$\begin{array}{l}\text { Number of peer-reviewed publications related to } \\
\text { POCUS }\end{array}$} \\
\hline $1-2$ & $1(10)$ \\
\hline $3-4$ & $5(50)$ \\
\hline$>10$ & $4(40)$ \\
\hline \multicolumn{2}{|l|}{$\begin{array}{l}\text { Completion of POCUS fellowship of 1-year duration or } \\
\text { more }\end{array}$} \\
\hline Yes & $6(60)$ \\
\hline No & $4(40)$ \\
\hline
\end{tabular}

* POCUS denotes point-of-care ultrasound.

\section{Expert Panel}

Our expert panel included 10 international POCUS experts who had at least 1 year of fellowship training in ultrasonography OR a 3-year track record of teaching and assessing POCUS AND a minimum of three peer-reviewed POCUS-related publications. The experts completed three rounds of the online survey between June 2019 and September 2019, where they were asked regarding specific minimal criteria and if a given item must or must not be present to meet minimal criteria. We defined consensus as agreement by at least $70 \%$ of the experts. ${ }^{23}$ Open-ended response options were also provided to capture missing items and additional comments. Items that did not reach consensus were included in subsequent rounds, which included new or revised items based on expert feedback from the previous round. Results from survey responses from prior rounds were provided to the participants in the form of the percentages of those who chose each option, as well as relevant comments that led to any item revisions.

\section{Results}

All 10 experts completed all rounds of the survey. Table 1 outlines the baseline characteristics of the experts.

\section{Round 1}

In round 1, of the 97 items considered, consensus was reached to include 50 items. For 18 items, consensus was reached for being NOT mandatory (Table 2). No consensus was reached for the remaining 29 items.

\section{Round 2}

Based on feedback from experts in round 1, two new questions were added to the 29 items that were without consensus from Round 1. The new questions were: (i) For a lung scan to be considered acceptable, one of the following lung patterns/ findings MUST be present deep to the pleural line: A-lines, $\mathrm{B}$ lines, consolidation, or pleural effusion and (ii) To rule out pneumothorax, the least gravitationally dependent areas of the chest MUST be scanned (e.g., anterior chest in a supine patient).

Of the 31 items considered in this round, 16 items reached consensus to be included, 4 reached consensus for NOT being mandatory, and 11 items did not reach consensus (Table 2).

\section{Round 3}

Round 3 included the 11 items from round 2 that did not meet consensus as well as one new item on the need to scan the posterolateral regions of the lungs for evidence of pneumonia/ consolidation. Of the 12 items considered, 8 reached consensus 
Table 2. Minimal Criteria For Internal Medicine Lung Ultrasound

\section{Minimal Criteria}

$\%$ agreement

\section{General Criteria}

$1 \quad$ For a lung scan to be considered acceptable, one of the following lung patterns/findings MUST be present deep to the pleural line: A-lines, B-lines, consolidation, or pleural effusion

2 Whenever possible, we recommend recording a minimum of a 6 -s cineloop

$3 \quad$ Using a lung preset is NOT mandatory if abnormalities can be visualized

$4 \quad$ Turning off tissue harmonic imaging is NOT mandatory if abnormalities can be visualized

Assessing for Pleural Sliding

Acceptable transducer(s)

5

6

Acceptable maneuvers to improve pleural sliding visualization

23 A still image of M-mode is acceptable to show the presence or absence of lung sliding

24 A B-mode still image is NOT acceptable to show the presence of lung sliding

A B-mode still image is NOT acceptable to show the absence of lung sliding

Minimal criteria for pleural sliding

Pleural line MUST be clearly visible

Pleural line MUST be clearly centered

Pleural line MUST be as echogenic as possible (by fanning)

To state that lung sliding is ABSENT, at least one rib MUST be visible

Showing two consecutive ribs is NOT mandatory

Lung zones MUST be labeled

If pleural sliding not seen, MUST use at least 1 maneuver

Change to a linear transducer

Adjust gain

Use M-mode

\section{Maneuvers deemed not mandatory}

\begin{tabular}{|l|l}
\hline 20 & \\
\hline 21 & \\
\hline 22 & \\
\hline & \\
\hline 23 & \\
\hline 24 & \\
\hline 25 & \\
\hline
\end{tabular}

Increasing frequency is NOT a mandatory maneuver

Moving the focus is NOT a mandatory maneuver

Using Doppler is NOT a mandatory maneuver

Assessing for Pleural Irregularities

\section{Acceptable transducer(s)}

$26 \quad$ Linear

$27 \quad$ Curvilinear

28 Microconvex

29 Phased array LEAST preferred

Minimal criteria for pleural irregularities

$30 \quad$ Pleural line MUST be clearly visible 


\section{General Criteria}

$31 \quad$ Pleural line MUST be clearly centered

\begin{tabular}{|l|l}
\hline 32 & Pleural line MUST be as echogenic as possible (by fanning)
\end{tabular}

\begin{tabular}{l|l}
\hline 33 & Lung zones MUST be labeled
\end{tabular}

$34 \quad$ Showing two consecutive ribs is NOT mandatory

\section{Assessing for Lung Point/Pneumothorax Assessment}

Acceptable transducer(s)

\section{Minimal criteria for lung point/pneumothorax assessment}

\begin{tabular}{|l|l}
\hline 39 & Pleural line MUST be clearly visible
\end{tabular}

\begin{tabular}{|l|l}
\hline 40 & Pleural line MUST be clearly centered
\end{tabular}

\begin{tabular}{l|l}
\hline 41 & Pleural line MUST be as echogenic as possible (by fanning)
\end{tabular}

\begin{tabular}{|l|l}
\hline 42 & Lung zones MUST be labeled
\end{tabular}

\begin{tabular}{lll}
\hline 43 & Must scan bilaterally to rule out a pneumothorax unless clinically not indicated (e.g., post-procedure)
\end{tabular}

\begin{tabular}{l|l}
\hline 44 & To rule out pneumothorax, the least gravitationally dependent areas of the chest MUST be scanned (e.g., anterior
\end{tabular} chest in a supine patient)

45 Lung point is NOT mandatory to diagnose pneumothorax

47 Showing two consecutive ribs is NOT mandatory

No consensus: At least one rib must be visible

\section{Still image archive for lung point/pneumothorax if cineloops not available}

47 A still image of $M$-mode is acceptable to show lung point

48 A B-mode still image is NOT acceptable to show lung point

\section{Assessing for B-Lines}

Acceptable transducer(s)

\begin{tabular}{l|l}
\hline 49 & Curvilinear
\end{tabular}

\begin{tabular}{|l|l}
\hline 50 & Phased array
\end{tabular}

\begin{tabular}{|l|l}
\hline 51 & Microconvex \\
\hline
\end{tabular}

\begin{tabular}{|l|l}
\hline 52 & Linear NOT acceptable*
\end{tabular}

\section{Minimal criteria for B-lines assessment}

\begin{tabular}{l|l}
\hline 53 & Pleural line MUST be clearly visible
\end{tabular}

\begin{tabular}{|l|l}
\hline 54 & Pleural line MUST be clearly centered \\
\hline
\end{tabular}

\begin{tabular}{|l|l}
\hline 55 & Pleural line MUST be as echogenic as possible (by fanning)
\end{tabular}

\begin{tabular}{l|l}
56 & At least one rib must be visible
\end{tabular}

\begin{tabular}{l|l}
\hline 57 & Lung zones MUST be labeled
\end{tabular}

\begin{tabular}{l|l}
58 & Must scan bilaterally to rule OUT pulmonary edema
\end{tabular}

\begin{tabular}{l|l}
\hline 59 & To rule OUT pulmonary edema, we encourage learners to scan a minimum of eight lung areas (four on each side)
\end{tabular} whenever possible

60 For the diagnosis of $B$-lines, we recommend a minimum depth of $10 \mathrm{~cm}$ below the pleural line be imaged

$61 \quad$ Showing two consecutive ribs is NOT mandatory 


\section{General Criteria}

Still image archive for B-lines if cineloops not available

62 A B-mode still image is acceptable to show positive B-lines

63 A B-mode still image is NOT acceptable to rule out presence of B-lines $90^{2}$

Assessing for Consolidation

Acceptable transducer(s)

Curvilinear

Phased array

Linear acceptable if consolidation can be visualized

Minimal criteria for consolidation

Pleural line MUST be clearly visible

Lung zones MUST be labeled

\begin{tabular}{|l|l}
\hline 70 & Must scan bilaterally to rule out a pneumonia \\
\hline
\end{tabular}

To look for pneumonia/consolidation, we encourage learners scan a minimum of eight lung areas (four on each side)

\begin{tabular}{l|l}
\hline 72 & To look for pneumonia/consolidation, posterolateral regions MUST be scanned
\end{tabular}

Pleural line being clearly centered is NOT mandatory

Pleural line being as echogenic as possible is NOT mandatory

If consolidation is visualized, having at least one rib visible is NOT mandatory

Showing two consecutive ribs is NOT mandatory

\section{Minimal criteria for basal zone consolidation}

MUST attempt to visualize the spine

78 Diaphragm MUST be visualized

Spleen or liver MUST be visualized

Lung zones MUST be labeled

Consolidation must show air bronchograms

Demonstrating the spleen/kidney or liver/kidney interface is NOT mandatory

83 Air bronchograms do NOT need to be dynamic

\section{Still image archive for consolidation if cineloops not available}

84 A B-mode still image is acceptable to show consolidation

\section{Assessing for Pleural Effusion}

Acceptable transducer(s)

85 Curvilinear

86 Phased array

$87 \quad$ Microconvex

88 Linear NOT acceptable*

Minimal criteria for pleural effusion

$89 \quad$ MUST attempt to visualize the spine

90 Diaphragm MUST be visualized

$91 \quad$ Spleen or liver MUST be visualized

92 Lung zones MUST be labeled

93 MUST scan bilaterally to rule out a pleural effusion

94 MUST scan a minimum of two zones to rule out pleural effusion 


\begin{tabular}{|l|l|l|}
\hline & General Criteria & \\
\hline 95 & To diagnose a pleural effusion, at least 25\% of the diaphragm MUST be visualized & $90^{3}$ \\
\hline 96 & Demonstrating the spleen/kidney or liver/kidney interface is NOT mandatory & $80^{2}$ \\
\hline 97 & Sinusoid sign is NOT mandatory & $70^{1}$ \\
\hline & Still image archive for pleural effusion if cineloops not available & $100^{1}$ \\
\hline 98 & A B-mode still image is acceptable to show presence of pleural effusion & \\
\hline
\end{tabular}

* Only nine experts responded to these questions.

Expert responses to items and the round in which the items achieved consensus are shown as superscript numbers

to be included, 2 reached consensus for NOT being mandatory, and 2 items remained without consensus (Table 2).

The final list of items is listed in Table 2 and included 74 mandatory items, 24 items that were considered NOT mandatory and 2 items that never reached consensus.

\section{Discussion}

In this study, we defined 74 consensus-based minimal criteria for key findings in Internal Medicine LUS. These criteria are both explicit and practical, taking into consideration potential equipment limitations such as commonly available transducers and machine functions (e.g., options for focus and transducer frequency modulation). It also takes into consideration the LUS findings in question. For example, while experts generally agreed that for most findings the pleural line should be visible, centered, made as echogenic as possible by fanning, and with at least one rib visible, in the presence of certain findings, such as an obvious consolidation, centering of the pleural line was not deemed mandatory (\#73) nor was it necessary that the pleural line itself be optimized (\#74). In another example (\#12), experts made it clear that if pleural sliding is visible, the presence of at least one rib may not be necessary. However, to state that lung sliding is ABSENT, at least one rib MUST be visible, so that the pleural line can be properly identified in such cases. Also, the group explicitly outlines image storage requirements. For example, while the presence of B-lines can be confirmed and documented with a still image (\#62), their absence cannot be so confirmed (\#63) due to the respiratory movement of B lines, making it possible to miss a positive finding with a still image. Such key information, while inherently known to LUS experts, has not been previously outlined and is vitally important for all learners and training programs to recognize.

There are some limitations to our study. First, a few of our items required significant revisions. For example, while 100\% of our experts agreed that a minimal depth must be achieved for the assessment of B-lines, (to avoid mistaking these for $\mathrm{z}$-lines), ${ }^{24}$ it was challenging to derive consensus on what this minimal depth should be, as it is partially dependent on the patient's body habitus (e.g., the more subcutaneous tissue depth present, the deeper the distance required). Ultimately, only by the third round were our experts able to achieve consensus once our revisions reflected this variability in subcutaneous tissue depth (\#60: a minimum of $10 \mathrm{~cm}$ required, starting from below the pleural line, rather than at the skin surface). We encountered similar wording issues when describing the amount of diaphragm required to be imaged in the assessment for pleural effusions. Rather than describing exactly which portions of the diaphragm were required to be visualized (medial vs lateral), experts were ultimately able to agree on the overall percentage of a diaphragm that must be visualized (\#95). Second, the online survey design did not allow for rich discussion amongst experts, and comments were limited to open text boxes only. However, despite this limitation, our experts were very engaged, and key comments from them led to significant wording revisions and additional items. Third, our minimal criteria are intended to be exactly that-minimal criteria. While using a lung preset and turning off tissue harmonic imaging should be performed whenever possible, if findings are seen, our group was willing to forego these settings (\#3, \#4). However, from a learner's perspective, it may still be prudent to be consistent in the use of an optimal preset or demonstrate the presence of both ribs using a curvilinear transducer whenever possible, even though these are not considered mandatory. Fourth, we did not perform a systematic review. However, our experts are true experts of LUS, including a number who participated in national and international consensus statements that did include an extensive literature strategy. ${ }^{8,11}$ Lastly, although not every expert answered every question in our survey, only two questions in all three rounds of the survey had missing responses.

In conclusion, our experts agreed upon 74 items required for meeting minimal criteria for LUS uses relevant to internal 
medicine, and 24 items were NOT required. In the teaching of LUS, adherence to these criteria is recommended.

\section{Statement of Contributions}

JD, VEN, ASL, and IWYM contributed to conception and design, and procurement of data; JD and IWYM contributed to the analysis of data, to the initial draft of the manuscript, and critical review and drafting of the manuscript.

\section{Statement of Funding}

This study was funded by the 2017 Canadian Society of Internal Medicine Education and Research Fund. The funder had no role in study design, data collection and analysis, decision to publish, or preparation of the manuscript.

\section{Conflict of Interest Statement for All Authors}

Dr. Ma is funded by the John A. Buchanan Chair of General Internal Medicine, University of Calgary.

Dr. Liteplo had received a grant from GE/EMF to perform ultrasound research, has consulted for Philips Healthcare, and is involved in ultrasound-related research sponsored by Fujifilm/ Sonosite unrelated to this research.

Dr. Buchanan is a clinical advisor for MEDO.Ai, in developing artificial-intelligence-based software for common point-of-care ultrasound applications.

Dr. Dversdal is employed part-time by Vave Health in the Chief Medical Officer's role. Primary contributions to this manuscript were all prior to this role.

Drs. Arishenkoff, Dumoulin, Desy, Noble, Liu, and Olszynski have no conflicts of interest to report.

\section{References}

1. American College of Physicians. ACP statement in support of point-of-care ultrasound in Internal Medicine [Internet]. 2018. Available from: https://www.acponline.org/ meetings-courses/focused-topics/point-of-care-ultrasound/ acp-statement-in-support-of-point-of-care-ultrasound-in-internal-medicine

2. Soni NJ, Schnobrich D, Matthews BK, et al. Point-of-care ultrasound for hospitalists: A position statement of the Society of Hospital Medicine. J Hosp Med. 2019;14:E1-6. http://dx.doi.org/10.12788/jhm.3079 | 10.12788/ jhm.3079

3. Ma IWY, Arishenkoff S, Wiseman J, et al. Internal Medicine point-of-care ultrasound curriculum: Consensus recommendations from the Canadian Internal Medicine Ultrasound (CIMUS) group. J Gen Intern Med. 2017;32:1052-7. http://dx.doi.org/10.1007/s11606-017-4071-5

4. Gargani L, Volpicelli G. How I do it: Lung ultrasound. Cardiovasc Ultrasound. 2014;12:25. http://dx.doi.org/10.1186/1476-7120-12-25

5. Perrone T, Maggi A, Sgarlata C, et al. Lung ultrasound in internal medicine: A bedside help to increase accuracy in the diagnosis of dyspnea. Eur J Intern Med. 2017;46:61-5. http://dx.doi.org/10.1016/j.ejim.2017.07.034
6. Filopei J, Siedenburg H, Rattner P, Fukaya E, Kory P. Impact of pocket ultrasound use by internal medicine housestaff in the diagnosis of dyspnea. J Hosp Med. 2014;9:594-7. http://dx.doi.org/10.1002/jhm.2219

7. Vitturi N, Soattin M, Allemand E, Simoni F, Realdi G. Thoracic ultrasonography: A new method for the work-up of patients with dyspnea. J Ultrasound. 2011;14:147-51. http://dx.doi.org/10.1016/j.jus.2011.06.009

8. Ma IWY, Hussain A, Wagner M, et al. Canadian Internal Medicine Ultrasound (CIMUS) expert consensus statement on the use of lung ultrasound for the assessment of medical inpatients with known or suspected coronavirus disease 2019. J Ultrasound Med. 2020. http://dx.doi.org/10.1002/ jum.15571

9. Ma IWY, Desy J, Woo MY, Kirkpatrick AW, Noble VE. Consensus-based expert development of critical items for direct observation of point-ofcare ultrasound skills. J Grad Med Educ. 2020;2:176-84. http://dx.doi. org/10.4300/JGME-D-19-00531.1

10. Ambasta A, Balan M, Mayette M, et al. Education indicators for internal medicine point-of-care ultrasound: A consensus report from the Canadian Internal Medicine Ultrasound (CIMUS) group. J Gen Intern Med. 2019;34:2123-9. http://dx.doi.org/10.1007/s11606-019-05124-1

11. Volpicelli G, Elbarbary M, Blaivas M, et al. International evidence-based recommendations for point-of-care lung ultrasound. Intens Care Med. 2012;38:577-91. http://dx.doi.org/10.1007/s00134-012-2513-4

12. Buda N, Kosiak W, Wełnicki M, et al. Recommendations for lung ultrasound in internal medicine. Diagnostics (Basel). 2020;10:597. http://dx.doi. org/10.3390/diagnostics10080597

13. Noble VE, Nelson BP. Manual of emergency and critical care ultrasound. New York: Cambridge University Press; 2011.

14. Soni NJ, Arntfield R, Kory P. Point-of-care ultrasound. Philadelphia, PA: Saunders; 2015

15. Lichtenstein DA. Whole body ultrasonography in the critically ill. New York, NY: Springer-Verlag; 2010.

16. Lichtenstein D, Meziere G, Biderman P. The comet-tail artifact. an ultrasound sign of alveolar-interstitial syndrome. Am J Respir Crit Care Med. 1997;156:1640-6. http://dx.doi.org/10.1164/ajrccm.156.5.96-07096

17. Lichtenstein D, Meziere G, Biderman P, Gepner A. The "lung point": An ultrasound sign specific to pneumothorax. Intensive Care Med. 2000;26:1434-40. http://dx.doi.org/10.1007/s001340000627

18. Lichtenstein D, Mezière G, Seitz J. The dynamic air bronchogram: A lung ultrasound sign of alveolar consolidation ruling out atelectasis. CHEST J. 2009;135:1421-5. http://dx.doi.org/10.1378/chest.08-2281

19. Lichtenstein DA, Lascols N, Prin S, Mezière G. The "lung pulse": An early ultrasound sign of complete atelectasis. Intensive Care Med. 2003;29:218792. http://dx.doi.org/10.1007/s00134-003-1930-9

20. Carmody KA, Moore CL, Feller-Kopman D. Handbook of critical care and emergency ultrasound. The McGraw-Hill Companies; 2011.

21. Levitov A, Dallas A, Slonim A. Bedside ultrasonography in clinical medicine. The McGraw-Hill Companies; 2011.

22. Levitov A, Mayo PH, Slonim AD. Critical care ultrasonography. New York, NY: The McGraw-Hill Companies; 2009.

23. Humphrey-Murto S, Varpio L, Gonsalves C, Wood TJ. Using consensus group methods such as Delphi and Nominal Group in medical education research. Med Teach. 2017;39:14-19. http://dx.doi.org/10.1080/01421 59X.2017.1245856

24. Lichtenstein DA. Current misconceptions in lung ultrasound: A short guide for experts. CHEST. 2019;156:21-5. http://dx.doi.org/10.1016/j. chest.2019.02.332 